\title{
The first series of laparoscopic radical cystectomies done in South Africa
}

\author{
Farzana Cassim, ${ }^{1}$ Sunil Sinha, ${ }^{2}$ Shameem Jaumdally, ${ }^{3}$ John Lazarus ${ }^{2}$ \\ ${ }^{1}$ Division of Urology Stellenbosch University \\ ${ }^{2}$ Division of Urology University of Cape Town \\ ${ }^{3}$ Division of Immunology, Institute of Infectious Disease and Molecular Medicine University of Cape Town
}

Corresponding author: Farzana Cassim (farzanac@icloud.com)

\begin{abstract}
Background: Radical cystectomy (RC) with extended lymphadenectomy and urinary diversion remains the standard of care for muscle-invasive urothelial carcinoma. Laparoscopic radical cystectomies (LRC) have been performed at Groote Schuur Hospital (GSH) since 2009. We aimed to audit our data regarding complications and oncological outcome and compare it to data obtained from patients undergoing open radical cystectomy (ORC) by the same surgeon since 2007.

Methods: All adult patients who underwent open and laparoscopic RC from 2007 to 2013 have been included in the study. Data on demographics, operative time, intraoperative blood loss, postoperative complications, margin positivity, and lymph nodes was obtained retrospectively by means of folder review.

Results: Thirty (30) patients who underwent LRC and 32 who underwent ORC were included in the study. Participants undergoing ORC experienced shorter operative duration (301 minutes versus 382 minutes; p-value < 0.0001), increased blood loss $(1376 \mathrm{ml}$ versus $779 \mathrm{ml}$; p-value $=0.0023)$ and transfusion requirement $(2$ units versus $0 ; \mathrm{p}$-value $=0.071)$ in contrast to LRC. Postoperative complications were more prevalent in the ORC arm compared to the LRC arm (61\% versus $43 \%)$. Patients with a past medical history were at higher risk of experiencing postoperative complications $(p$-value $=0.04$; Risk Ratio: 1.6). Margin positivity was comparable between the two arms. A higher number of nodes was sampled by the laparoscopic technique in this study (overall p-value $=0.07$ ).

Conclusion: Laparoscopic RC is associated with longer operative times, decreased blood loss, and equivalent oncological outcomes when compared to ORC. Laparoscopic RC is a feasible option in our setting.
\end{abstract}

\section{Introduction}

Worldwide, bladder cancer (BC) is the 9th most prevalent cancer according to the World Cancer Research Fund International in 2012. ${ }^{1}$ Cancer of the bladder is the 7 th most common cause of death in South African males, and the 16th most common cause of death in South African females according to the National Cancer Registry statistics from 2014. ${ }^{2}$

The predominant type of $\mathrm{BC}$ is urothelial carcinoma (UC). ${ }^{3}$ Of all the $\mathrm{BC}$ diagnosed, approximately $70 \%$ are not muscle-invasive and are managed endoscopically. ${ }^{4}$ The risk of progression of non-muscle invasive urothelial carcinoma in 5 years varies from $0.8-45 \%$, depending on the risk profile of the patient and tumour grade. ${ }^{4}$ In patients with superficial high-grade tumours, the risk of progression at 5 years is $54 \%$ without Bacillus Calmette-Guérrin (BCG) instillation, and $10-20 \%$ with BCG instillation (induction and maintenance therapy). ${ }^{4}$ Thirty percent of patients require more radical therapy from the time of diagnosis for muscle-invasive disease. ${ }^{4,5}$ This includes radical surgery, radiotherapy with curative intent, or bladder-preserving protocols..$^{3-5}$
Radical cystectomy (RC) with extended lymph node dissection (ELND) and urinary diversion (UD) is the standard of care for treatment of localised muscle-invasive BC. ${ }^{3,4}$ Radical cystectomy has traditionally been performed by open surgery. However, since the advent of laparoscopy and robotic-assisted LRC, there has been a worldwide shift towards minimally invasive surgical procedures.

According to the available literature, it appears as though there is no option that provides oncological superiority to another in terms of surgical technique used. ${ }^{4}$ There does seem to be improved outcomes with regard to blood transfusion requirements, incidence and duration of ileus, wound complications and time to discharge in the laparoscopic group..$^{6-9}$ Duration of surgery is shorter in the open surgery group of patients. ${ }^{8,9}$

Our institution, a tertiary centre serving a mainly indigent population, is currently one of few centres in South Africa (SA) performing RC using laparoscopy. We aim to present our data comparing all open and laparoscopic radical cystectomies performed by a single surgeon in order to highlight the possible advantages of performing LRC. 


\section{Patients and methods}

\section{Data Collection}

Approval was obtained from the Human Research Ethics Committee of the Faculty of Health Sciences of the University of Cape Town. The folders of all patients undergoing ORC and LRC performed at our centre from 2007-2013 were requested, and those available were retrospectively reviewed. Only patients with complete data sets were included in the study.

The data that was collected included demographics, so as to confirm that the cohorts being compared were age-matched. We also compared the prevalence of comorbidities in the two groups. Data pertaining to operative duration (including UD) was reviewed, as well as peri- and postoperative complications. Complications were documented according to the Clavien-Dindo Classification. Specific complications that were examined more in-depth included intraoperative blood loss, wound complications, postoperative ileus, as well as data on oncological control, such as number of lymph nodes obtained, number of positive lymph nodes, and margin positivity. Pre- and postoperative staging data were compared.

\section{Surgical Technique}

The basic principles of surgery for the treatment of bladder cancer were applied to both surgical methods used. This included a modified ELND (up to the level of the midcommon iliac vessels, excluding presacral lymph node sampling), removal of the bladder (and all macroscopic tumour around the bladder), removal of the uterus in females or the prostate and seminal vesicles in males, with or without a urethrectomy. ${ }^{3}$ Extra-corporeal UD was then performed by way of an ileal conduit (as per surgeon preference).

Positioning differs for ORC as compared to LRC. During ORC, the patient is placed in Lloyd-Davis (low lithotomy) position, with mild Trendelenburg (head down) positioning to facilitate the surgery. During LRC, the patient is also placed in Lloyd-Davis position, but the patient is required to be placed in steep Trendelenburg position, which may be associated with increased risk of intraoperative complications. These include raised airway pressure with atelectasis, cerebral and facial oedema, raised intra-ocular pressure, and traction on lower limbs with neuropraxia. ${ }^{10,11}$ No documented cases of these complications were noted in this cohort of patients.

\footnotetext{
Statistical Analysis

Continuous variables were reported using medians and interquartile ranges. Categorical variables were reported as a percentage of the total and proportion of the main subset. Continuous variables were compared by Mann-Whitney U test. Categorical variables were compared between groups by Fisher's exact test. Spearman's correlation coefficients were calculated to assess potential associations between continuous variables. No adjustment for multiple comparisons was used, given the small sample size and the hypothesisgenerating nature of the study. Two-sided p-values $<0.05$ were considered significant. All analyses were performed using GraphPad Prism v5.0 (La Jolla, CA).
}

\section{Results}

\section{Participants' Characteristics}

Seventy-five patients underwent RC from January 2007 to February 2013 at GSH, Cape Town. Of these 75, 39 were operated on using laparoscopic technique and 35 underwent ORC. Thirty (30) patients from the laparoscopic arm were included as participants in this study while 32 from the open arm were included. Thirteen (13) patients were excluded based on the lack of data. One patient was excluded as the patient was converted from LRC to ORC relatively early in the procedure. A significantly higher proportion of men underwent laparoscopic surgery when compared to women ( $83.3 \%$ versus $16.7 \%$ respectively, $\mathrm{p}=0.02)$, while the gender balance was approaching symmetry in the open arm $(56.3 \%$ male versus $43.8 \%$ female). The mean age of participants included across the two arms did not differ (mean of 61 for laparoscopic surgery and 60 for open surgery). Moreover, when classified according to gender and comparing across the two arms, there was no difference in age observed.

Of the 30 participants included in the laparoscopic arm, $45 \%$ presented with comorbidities, while $55 \%$ of patients in the open arm had pre-existing conditions. These were mainly medical in nature. Two patients in the open group were known with ischaemic heart disease, as opposed to only 1 patient in the laparoscopic group. One patient in each arm had suffered a prior cerebrovascular accident. The leading comorbidity was hypertension.

\section{Clinical Staging}

On preoperative screening (ultrasound), there was no difference in the prevalence of hydronephrosis. Every patient referred for RC underwent an examination under anaesthesia (EUA) and a transurethral resection of the bladder tumour. The EUA findings suggest that patients with higher stage disease were more likely to undergo open surgery as opposed to laparoscopic (59\% having a palpable mass on EUA in the open group versus $36 \%$ in the laparoscopic group, $\mathrm{p}=0.08$ ).

\section{Intraoperative Parameters}

The duration of the two procedures differed significantly with a mean duration of 382 minutes for the laparoscopic intervention as opposed to a mean of 301 minutes for open surgery. The duration documented included extra-corporeal formation of an ileal conduit for all cases.

A significantly higher median volume of blood loss was encountered during the open intervention when compared to the laparoscopic one $(1376 \mathrm{ml}$ for open versus $779 \mathrm{ml}$ for laparoscopic; Figure 1A). Of these patients, 38\% of participants in the laparoscopic arm required transfusion compared to $64 \%$ of patients undergoing open surgery $(p=0.10)$. The median number of units required for transfusion was 0 for the laparoscopic arm compared to 2 for the open arm (Figure 1B). There was no association between the detection of a mass at EUA and intraoperative bleeding.

Three patients experienced intraoperative complications that were not haemorrhagic in nature. One participant from 


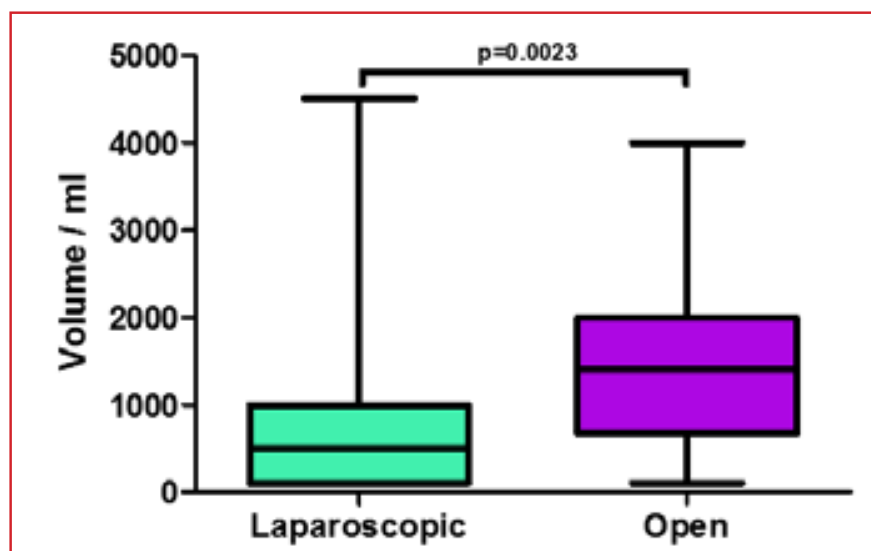

Figure 1A: Volume of blood loss during intervention.

the laparoscopic group experienced acidosis secondary to hypoventilation and hypocarbia requiring postoperative intubation. A participant from the open group experienced rectal injury requiring colostomy. One patient in the laparoscopic group also experienced a rectal injury, which was repaired primarily intraoperatively and required no further intervention. On clinical staging, it was observed that significantly more participants were staged as T1/T2 disease in the laparoscopic arm compared to the open arm $(87 \%$ versus $43 \%, \mathrm{p}<0.0001)$.

\section{Postoperative complications}

More participants reported postoperative complications in the open group when compared to the laparoscopic group $(61 \%$ for the open group compared to $43 \%$ for the laparoscopic

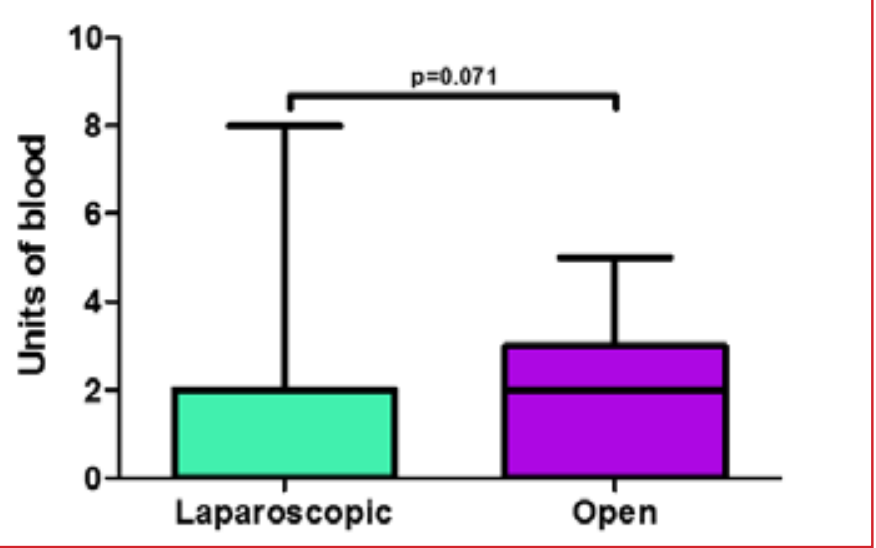

B: Units of blood required for transfusion

group). This difference was however not statistically significant. This trend was confirmed when the degree of complications (Clavien) was compared between the two arms (Figure 2).

Participants who have reported a past medical history were significantly more at risk (risk ratio of 1.6) of experiencing postoperative complications $(48 \%$ in participants with no medical history as compared to $77 \%$ for participants with a past medical history; $\mathrm{p}=0.04$ ). When investigating further into the nature of the postoperative complications, it was observed that the only complication that differed in proportion between the two arms were those related to wound complications ( $18 \%$ for laparoscopic group versus $44 \%$ for the open group). The predominant wound complication was sepsis. Ileus was identified as the main gastrointestinal complication.

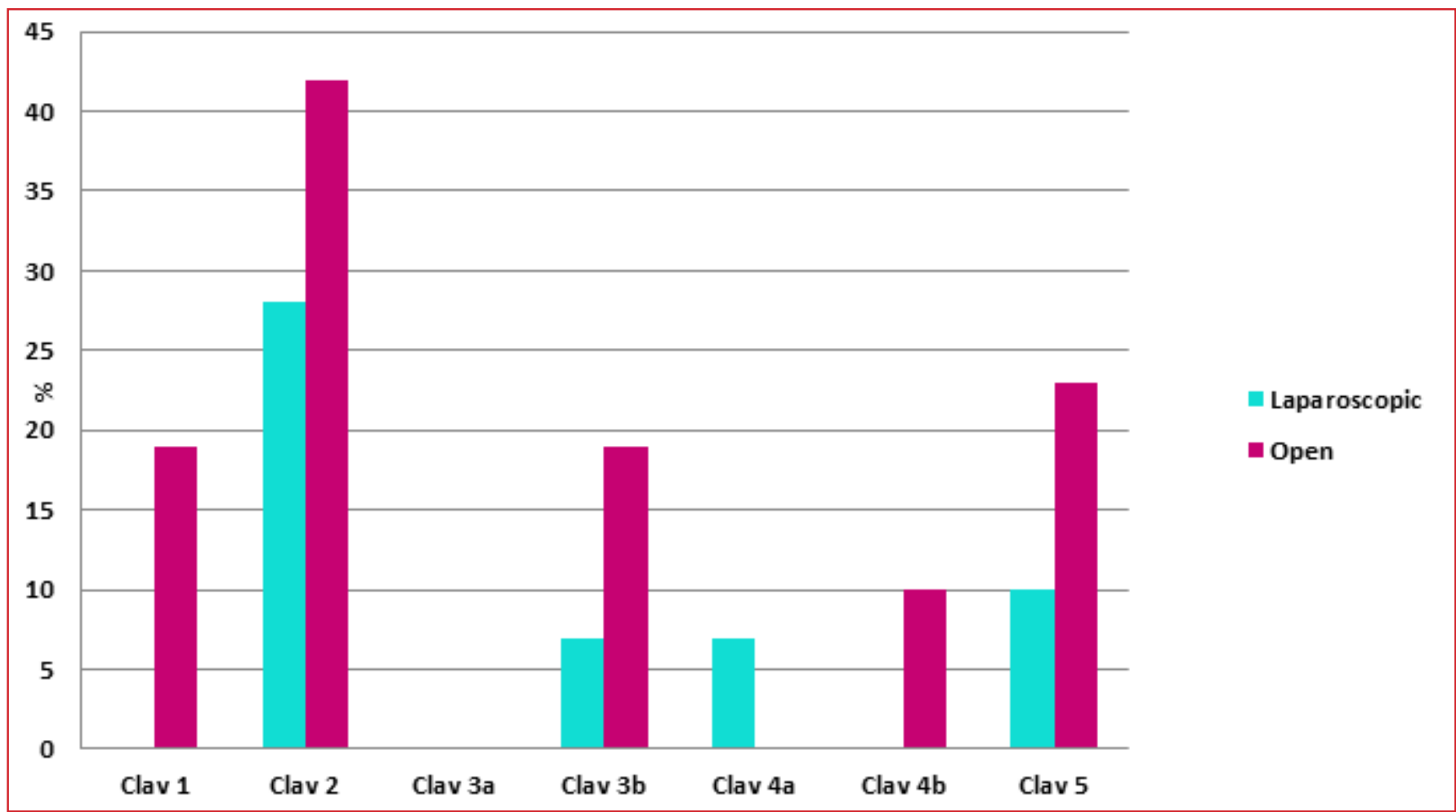

Figure 2: Clavien classifications across two arms 


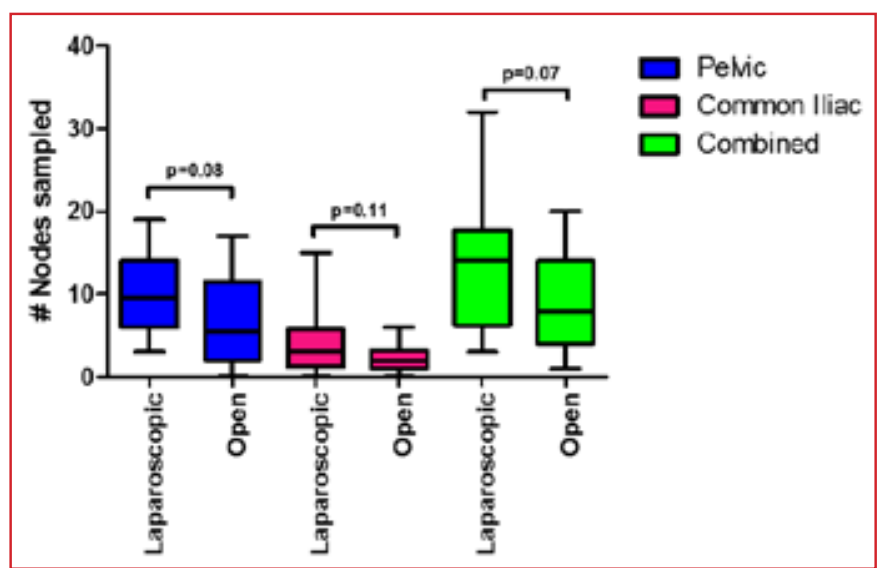

Figure 3: Number of lymph nodes sampled during intervention

One patient in the open group developed an anastomotic stricture requiring revision of the ileal conduit. This patient also experienced a rectal injury requiring a diverting colostomy. One patient in the laparoscopic group required a relook laparotomy for an anastomotic leak.

There was no association between age of participants and the incidence of postoperative complications, irrespective of the type of procedure undergone. Participants with a mass on EUA were more likely to experience overall postoperative complications $(60 \%)$ when compared to participants with no mass detected on EUA (47\%), although this is not the case for wound complications specifically. When looking at the prevalence of postoperative complications among participants who experienced intraoperative complications compared to those who did not experience intraoperative complications, no difference was observed. Following the difference in clinical staging found when comparing the 2 arms, the pathological staging of the two groups was compared revealing no difference between the two arms.

\section{Oncological outcomes}

When comparing margin positivity in the two groups no difference was found when looking at clinical staging. However, a strong trend emerged where all positive margins were only encountered for stages $\mathrm{T} 3$ and T4 disease. The surgical modality used did not impact on this.

A trend was observed when comparing the number of lymph nodes sampled using the two techniques whereby a higher number of nodes was most often sampled for using the laparoscopic option in this study (Figure 3).

\section{Discussion}

Radical cystectomy is well established as the standard of care for $\mathrm{BC}$, with ORC as the gold standard., ${ }^{4,5}$ Minimally invasive techniques, including LRC and robotic-assisted LRC are being described as feasible treatment options on a more regular basis. ${ }^{8,9,12}$ The proposed reasons for this trend towards minimally invasive surgery include decreased blood loss, with decreased postoperative complications and equivalent oncological outcomes. ${ }^{7,14}$
Concerns surrounding LRC as a truly equivalent technique when compared to ORC have been raised due to the lack of good quality evidence to support this. ${ }^{8,13}$ Comparisons involving unmatched cohorts in terms of the stage of the disease process pose the largest problem when studying the rate of complications, as well as oncological outcomes between the two groups. ${ }^{13}$

Our data is comparable to that which has been previously published. ${ }^{13-15}$ We reviewed two age-matched groups of patients undergoing ORC and LRC performed by a single surgeon at our centre between January 2007 and February 2013. The demographics were similar between the 2 groups, except for the significantly higher proportion of men who underwent laparoscopic surgery when compared to the number of women undergoing LRC. Due to the retrospective nature of the study, it is difficult to assess the reasons for this difference. A selection bias exists as patients with a lower clinical T-stage were selected by the operating surgeon for LRC in order to facilitate the learning curve for this procedure. The difference does, however, compare with worldwide studies where more males than females underwent RC. ${ }^{6,7,9}$ There was no association found between age or gender and the prevalence of complications. There was also no statistical difference in the prevalence or types of pre-existing co-morbidities between the 2 groups. A difference in clinical staging was observed between the 2 arms, with patients undergoing open surgery being more likely to have a higher clinical stage (59\% clinical T3 based on EUA in the open arm versus $36 \%$; $p=0.08$ ). This stage bias has been described in multiple previous trials, and is one of the criticisms of studies claiming the superiority of minimally invasive surgery for bladder cancer. ${ }^{8,13}$

Operative time was significantly less with open surgery, as has been seen in multiple previous studies. ${ }^{6,16-20}$ Bleeding was the commonest intraoperative complication. Blood loss and rates of transfusion were significantly lower in the laparoscopic group in our study. This is in line with the current data that is available when comparing these 2 modalities. ${ }^{16-21}$ The volume of blood loss was not increased by higher stage disease.

Selection bias has been stated to be significant when comparing patients undergoing LRC versus ORC. Our results were not dissimilar, in that significantly more patients undergoing laparoscopic RC were Stage T1/T2 disease. However, our findings suggest that this does not necessarily impact on outcomes. The blood loss seen across all participants was greater in the patients with T1/T2 disease as compared to patients with T3/T4 disease. There was no other association with bleeding that could be found to explain this phenomenon.

The predominant postoperative complication globally was ileus, while wound complications were most prevalent in the patients undergoing ORC. None of these differences were statistically significant. Studies from high-volume centres also found these differences, but in these studies, the differences were indeed found to be statistically significant. ${ }^{9,18-20,22}$ The overall prevalence of postoperative complications in both groups in our study was higher in patients with a palpable mass 
on examination under anaesthesia, as is to be expected. There was no statistically significant correlation between increased age and increased risk for postoperative complications. This contradicts the findings by Clarke et al. which showed that age impacts on postoperative outcomes. ${ }^{23}$

The overall survival rates are comparable in the 2 groups. However, given the relatively short follow-up period (median: 5 years ORC; 3 years LRC) and the small numbers of participants, it is not possible to accurately comment on overall survival. In terms of early postoperative mortality, it is apparent that the 2 groups have similar 1-month postoperative mortality rates. At least $50 \%$ of the patients who died from both groups had palliative cystectomies for advanced disease. Other causes of death included myocardial infarction in patients with pre-existing cardiac dysfunction, sudden death from presumed pulmonary emboli, as well as septic shock. All patients were managed as part of a multi-disciplinary team.

In terms of oncological outcomes, the surrogate markers that were used included positive surgical margins and lymph node yield. Positive margins are clearly undesirable due to incomplete oncological control, as well as the increased risk of early local recurrence. The number of lymph nodes sampled, irrespective of status, has been shown to impact on prognosis and overall survival., ${ }^{27,28}$ These markers have been used in multiple studies previously, and our findings are in line with those noted in these studies. ${ }^{24-28}$

Multiple studies have found that the oncological outcomes using the above-mentioned criteria are similar between the 2 surgical modalities. ${ }^{7-9,15,16}$

One limitation of this study is the retrospective nature of the study. Unfortunately, a large number of folders were not accessible for various reasons. Due to this study design as well as the number of patients lost to follow-up, it is not possible to comment adequately on survival outcomes, but from using the available data alone, it is apparent that these outcomes are not affected by the surgical modality used. Another limitation noted is a deficiency in standardised documentation of patient progress reports in terms of time to first meal and time to passing stool. Being a referral centre for advanced BC serving a wide population distribution, several patients were seen at outlying hospitals for follow-up, thereby affecting the number and exact timing of documentation of postoperative complications. Another limitation is the relatively small numbers of participants in the study. The discrepancy in follow-up times between the 2 groups, whereby the open group has a longer follow-up time (median: 5 years) when compared to the laparoscopic group (median: 3 years), is an additional limitation. Also, due to the nature of the disease process, and the stage at which patients present in our setting, it is difficult to always compare these cases without confounding factors being present. Given that minimally invasive surgery has been developed to improve postoperative pain and shorten hospital stay, the lack of these data is also a limitation.

The benefit, however, of assessing data from a single surgeon eliminates possible differences in surgical technique that may be considered to be the cause of differences in complications and outcomes. Although generalisability is not possible, for the purposes of this study, it is possible to show feasibility of one surgeon performing both techniques with similar outcomes.

\section{Conclusion}

With this study documenting the early experience of LRC in our centre we are able to conclude that LRC is associated with a decreased need for blood transfusions. Open RC is associated with shorter operative time. The two options are similar in terms of complication rates and oncological outcomes. Laparoscopic RC is therefore a feasible option in our setting.

Given the number of patients who have subsequently undergone LRC at GSH since the completion of this study, it is clear that the number of potential participants for a future study may allow for more definitive statements to be made regarding which modality may provide better outcomes. The volume of RC currently being performed at our centre may allow for a prospective study design, thereby producing more robust evidence, which may guide practice in the future.

\section{Conflict of interest}

None

\section{REFERENCES}

1. Ferlay J, Soerjomataram I, Ervik M, et al. GLOBOCAN 2012 v1.0, Cancer Incidence and Mortality Worldwide: IARC CancerBase No. 11 [Internet]. Lyon, France: International Agency for Research on Cancer; 2013. Available from: http:// globocan.iarc.fr, accessed on 13/12/2013

2. National Cancer Registry 2014 [accessed on 13/09/2018] Available from: http://www.nicd.ac.za/wp-content/ uploads/2017/03/2014-NCR-tables-1.pdf

3. Wood Jr. DP. Urothelial Tumours of the Bladder. CampbellWalsh Urology; 10th Edition; Kavoussi LR, Novick AC, Partin AW, et al. Elsevier Saunders 2012; Vol. 3; 80: pp. 2311-2332.

4. Witjes JA, Comperat E, Cowan NC, et al. Guidelines on MuscleInvasive and Metastatic Bladder Cancer; EAU Guidelines 2014.

5. Lerner SP: Bladder Cancer. The American Urological Association Educational Review Manual in Urology 2012 (4th Ed); Chapter 18: pp. 549-83.

6. Zheng W, Li X, Song G, et al. Comparison of laparoscopic and open cystectomy for bladder cancer: A single centre of 110 cases report. Transl Androl Urol. 2012;1:4-8. Available from: https://www.ncbi.nlm.nih.gov/pmc/articles/PMC4713212

7. Guillotreau J, Game X, Mouzin M, et al. Radical cystectomy for bladder cancer: Morbidity of laparoscopic versus open surgery. J Urol. Feb 2009;181:554-9. doi: 10.1016/j.juro.2008

8. Aboumarzouk OM, Hughes O, Narahari K, et al. Safety and feasibility of Laparoscopic Radical Cystectomy for the treatment of bladder cancer. J Endourol. Sep 2013;27(9):108395. doi: 10.1089/end.2013.0084

9. Cathelineau X, Arroyo C, Rozet F, et al. Laparoscopic Assisted Radical Cystectomy: The Montsouris Experience after 84 Cases. Eur Urol. 2005;47:780-4. Available from: www. europeanurology.com/article/S0302-2838(05)00201-0/fulltext

10. Ackerman RS, Cohen JB, Garcia Getting RE, Patel SY. Are you seeing this: the impact of steep Trendelenburg position 
during robot-assisted laparoscopic radical prostatectomy on intraocular pressure: a brief review of the literature. J Robot Surg. Jul 2018; e-pub ahead of print.

11. Choi ES, Oh AY, In CB, Ryu JH, Jeon YT, Kim HG. Effects of recruitment manoeuvre on perioperative pulmonary complications in patients undergoing robotic assisted radical prostatectomy: A randomised single-blinded trial. PLoS ONE. 2017;12(9):e0183311. Available from: https://doi.org/10.1371/ journal.pone. 0183311

12. Simonato A, Gregori A, Lissiani A, et al. Laparoscopic radical cystoprostatectomy: Our experience in a consecutive series of 10 patients with a 3 years follow-up. Eur Urol. 2005;47:78592. Available from: www.europeanurology.com/article/S03022838(05)00082-5/fulltext

13. Puppo P, Naselli A. Laparoscopic radical cystectomy: Where do we stand? Arch Esp Urol. 2010;63(7):508-19.

14. Cohen SA, Mirheydar HS, Parsons K, et al. Minimally Invasive Cystectomy Is Associated With Improved Perioperative Patient Safety Outcomes Compared With Open Cystectomy in a National Cohort. Urology. 2014;84:314-20.

15. Ha US, Kim SI, Kim SJ, et al. Laparoscopic versus open radical cystectomy for the management of bladder cancer: Mid-term oncological outcome. Int J Urol. 2010;17:55-61.

16. Gillion N, Evanguelos X, Xavier D, et al. Mid-term oncological control after laparoscopic radical cystectomy in men: a singlecentre experience. BJUI. 2011;108:1180-4. doi: 10.1111/j.1464410X.2010.10054.x/full

17. Tang K, Li H, Xia D, et al. Laparoscopic versus open radical cystectomy in bladder cancer: a systematic review and metaanalysis of comparative studies; PLoS ONE. 2014;9(5):e95667. doi: 10.1371/journal.pone.0095667

18. Gerullis H, Kuemmel C, Popkin G. Laparoscopic Cystectomy with Extracorporeal-Assisted Urinary Diversion: Experience with 34 Patients. Eur Urol. 2007;51:193-8.

19. Khan MS, Challacombe B, Elhage O, et al. A dual-centre, cohort comparison of open, laparoscopic and robotic-assisted radical cystectomy. Int J Clin Pract. 2012;66(7):656-62. doi: 10.1111/j.1742-1241.2011.02888.x

20. Porpiglia F, Renard J, Billia M, et al. Open versus Laparoscopy-
Assisted Radical Cystectomy: Results of a Prospective Study. J Endourol. March 2007;21(3):325-32. Available from: https:// doi.org/10.1089/end

21. Hemal AK, Kolla SB. Comparison of Laparoscopic and Open Radical Cystoprostatectomy for Localized Bladder Cancer With 3-Year Oncological Follow-up: A Single Surgeon Experience. J Urol. 2007;178:2340-3. doi: 10.1089/089277903772036271

22. Chang SS, Cookson MS, Baumgartner RG, et al. Analysis of early complications after radical cystectomy: results of a collaborative care pathway. J Urol. May 2002;167:20126. Available from: https://www.ncbi.nlm.nih.gov/ pubmed/11956429

23. Clark PE, Stein JP, Groshen SG, et al. Radical cystectomy in the elderly: comparison of clinical outcomes between younger and older patients. Cancer. Jul 2005;104(1):36-43. Available from: https://www.ncbi.nlm.nih.gov/pubmed/15912515

24. Shariat SF, Rink M, Ehdaie B, et al. Pathologic Nodal Staging Score for Bladder Cancer: A Decision Tool for Adjuvant Therapy After Radical Cystectomy. Eur Urol. 2013;63:371-8. Available from: http://dx.doi.org/10.1016/j.eururo.2012.06.008

25. Dotan ZA, Kavanagh K, Yossepowitch O, et al. Positive surgical margins in soft tissue following radical cystectomy for bladder cancer and cancer specific survival. J Urol. 2007;178:2308-13. Available from: https://www.ncbi.nlm.nih. gov/pubmed/17936804

26. Stein JP, Quek ML, Skinner DG. Lymphadenectomy for invasive bladder cancer II: technical aspects and prognostic factors. BJUI. 2006;97:232-7. doi:10.1111/j.1464-410X.2006.05901.x

27. Herr HW, Bochner BH, Dalbagni G, et al. Impact of the number of lymph nodes retrieved on outcome in patients with muscle invasive bladder cancer. J Urol. 2002;167:1295-8. Available from: https://www.ncbi.nlm.nih.gov/pubmed/11832716

28. Leissner J, Hohenfellner R, Thuroff JW, et al. Lymphadenectomy in patients with transitional cell carcinoma of the urinary bladder: significance for staging and prognosis. BJU Int. 2000;85:817-23. Available from: https://www.ncbi.nlm.nih.gov/ pubmed/10792159 